\title{
Investigating the bundle compliance of IVIG delivery in the Greater Manchester Neuroscience centre
}

\author{
Neena Patel, Amanda Woodall, David Gow \\ Salford Royal Hospital, Salford, UK
}

\begin{abstract}
Salford Royal Hospital is one of the largest users of IVIG for chronic neurological illnesses within the UK. The majority of patients are being treated for chronic inflammatory polyneuropathy and multifocal motor neuropathy. We hypothesised that the components of care being delivered to these patients differed to our stated standard of care (IVIG care bundle). We performed a service review exercise to identify shortcomings and improve quality of patient care. The aim was to measure overall bundle compliance being delivered to 75 patients with a view to improving the overall quality of care being delivered in the future. A retrospective case note study was carried out to measure compliance with the 17 areas of care, which constituted the IVIG bundle. Nine areas of care were being delivered to all 75 patients. This meant that all patients were receiving three monthly bloods, a documented cannula pathway, a filed prescription, a medical assessment, and the correct follow-up. Not all patients had a filed consent form, ECG or HAT assessment and an even smaller number of patients had a documented calculation for the amount of IVIG that needed to be given and few had a serum save. No patient in the group was receiving the intended complete bundle of care.
\end{abstract}

The results led to the development of an electronic treatment dashboard for the delivery of chronic IVIG therapy to this group. A re-audit has shown that rates of individual areas of care being delivered has increased markedly but overall compliance has only increased a slightly due to a lack of serum saves for patients.

\section{Problem}

Two main problems were identified. The first was to establish areas of care that should make up the bundle of care to be delivered as this was not made immediately clear at the start of the project. The second problem was to identify how many patients were in fact receiving the proposed areas of care with a view to looking at overall bundle compliance. Once the answer to both of these questions became clear, we wanted to work towards implementing a system to improve the overall bundle compliance for this group of patients.

\section{Background}

Intravenous immunoglobulin is a blood product first used over 30 years ago as a treatment for immunodeficiency disorders. Since then a mono metric suspension of IgG was created as a treatment for other ailments such as autoimmune diseases and acute infections (1).

Within the neurological speciality, IVIG has been licensed to treat many conditions but is used mainly to treat chronic inflammatory demyelinating polyradiculopathy (CIDP) and multifocal motor neuropathy (MMN) at Salford Royal hospital.

IVIG is a huge cost to the NHS at $£ 32$ per gram. Each patient being treated will require 2 grams/kilogram of the drug for it's effects to take place(2). A typical patient being treated at Salford Royal hospital will need treatment every 4 to 6 weeks, making this a costly treatment to the department as a whole.

The use of this treatment is strictly monitored by the department of health largely due to it's expense to the NHS as a whole but also because the NHS has experienced shortages of IVIG in the past (3).

\section{Baseline Measurement}

Senior staff involved in the delivery of IVIG first met to decide what areas of care they expected the service to deliver to these patients. Based on areas of care identified from that meeting, a pro-forma was designed to see how many patients were in fact receiving the hypothesised bundle of care.

A retrospective audit was then carried out to look at the areas of care being delivered to 75 patients being treated with IVIG. Case notes were pulled from the department both in their electronic and paper form. The data collected looked at care being delivered before, during, and after treatment. It looked at the safety of delivering this blood product as well as the quality of the care being received. Before the treatment was commenced, the intended bundle meant that each patient should have received a pretreatment ECG, a pre-treatment serum save, and a viable consent form. The department was also required to provide a department of health form for each patient being treated so that the department of health could monitor the uses of the product. For the actual treatment to be delivered, a patient should have had a hospital acquired thrombosis assessment, a cannula pathway, a documented calculation for running rates dependent on patient 
weight, and a paper prescription. Post treatment, patients required three monthly bloods, a general medical assessment with an in depth neurological review, and discharge documentation. Patients were also required to be followed up every 6-12 months once they are in the treatment programme and should have a documented future appointment before leaving the hospital.

Results from the audit showed that some areas of care were running at $100 \%$ as illustrated by the graph. A documented calculation for running rates and Romberg's test were lacking for over $80 \%$ of the group.

A small number didn't receive a pre-treatment ECG or an observed walk with $95 \%$ and $81 \%$ receiving them respectively. Not one patient was receiving every aspect of care intended, resulting in a bundle compliance of $0 \%$.

See supplementary file: ds2195.docx - "ivig graph"

\section{Design}

Upon recognising that the quality of the service was inadequate, the help of the IT department was enlisted to design an electronic treatment dashboard to monitor compliance of the care bundle.

The programme has now been integrated into the main electronic patient records. This enables the dashboard to automatically provide triggers to alert health care professionals when a component of the care bundle has been overlooked and helps to avoid human error.

\section{Strategy}

In the first instance, nursing and medical staff needed to be educated on what the components of the care bundle were and what areas they were expected to be delivering. A meeting was organised to ensure the entire team was clear on the contents of the bundle and during which stage of treatment a patient should be receiving the component.

Once the team had been educated on the basics of the care bundle, they were trained up on how to use the electronic trigger board. This was easily demonstrated as all of the clinical staff at Salford Royal hospital use electronic patient records and so a small implementation such as this was simple for staff to follow and use.

\section{Results}

All patients are now required to have a hepatitis $B$ and $C$ test pretreatment. This is a new addition to the original bundle of care. The electronic trigger board has ensured that all patients receive this test.

Analysis of the current delivery of IVIG shows that all the areas of care previously running at $100 \%$ are still running at $100 \%$.

Some areas of the care bundle have now been adjusted dependent on a patient's tailored needs. Only patients requiring a hospital stay for their treatment treated require a hospital acquired thrombosis assessment now. $100 \%$ of inpatients are currently receiving this and this is documented as part of the patient's electronic notes which are separate from the trigger board.

Romberg's assessment is now only required for patients not being treated for chronic inflammatory demyelinating polyneuropathy and is not part of the care bundle for all patients. Only $21.3 \%$ of our population requires this test now, and that has been recognised in the new model of care.

All patients now have a pre-treatment ECG a and a documented walk as part of the medical assessment. The trigger board has allowed an electronic record to be kept for the calculation of running rates for all patients and has led to all patients having this documentation as part of it. This is a marked improvement on the previous audit which only showed $13 \%$ of the group having this.

The figures for current patients having a consent form has remained the same for the population looked at. All new patients have a filed consent form and long term patients without consent forms will be consented at the next review.

An area of care not improved is the number of patients receiving a serum save. These figures have remained the same because there is not enough room in the hospital laboratory to keep every patient's sample. It is estimated now that bundle compliance is certainly above $0 \%$ but is no more than $20 \%$ for the entire group based on the small number of patients receiving a serum save.

See supplementary file: ds2192.docx - "ivig trigger board"

\section{Lessons and Limitations}

Almost every IVIG patient notes were looked at for this project but a few were unavailable at the time. The numbers of patients left out of the study were only five, and so though having their information as part of our research may have skewed the results; the differences would have been minor.

We hypothesised that calculations for running rates were calculated but not documented in the paper or electronic notes, which affected our compliance in that area. It could have also been the case that specifics of the medical examination were also done but not documented affecting these results also.

The difficulties of this project became apparent at the start when the pro-forma was designed. This was because there was no documented national or trust guideline to aid what components should make up the care bundle. The components were based on what seniors in the delivering team had hypothesised should be part of the bundle based on their clinical inclination.

At the start of the project it was unknown whether other medical staff in the department were aware of what components of care needed to be delivered. The $0 \%$ bundle compliance figure indicates that this was a problem. The important lesson learnt from this 


\section{BMJ Quality Improvement Reports}

project was to ensure that the entire team delivering IVIG were clear on the standard of service that should be provided to this group.

Whether this is the correct and definitive care bundle for patients receiving IVIG is unknown because there are no national guidelines to aid clinicians. This meant that we were unable to compare our care bundle and bundle compliance figures with other trusts across the UK to ensure that the bundle was entirely correct.

The main limitation to this project is the lack of serum saves for each patient. Bundle compliance will not increase by any amount unless room is found for serum saves to be stored.

\section{Conclusion}

At the end of this project the entire team delivering IVIG were clear on what the care bundle was in the first instance, meaning that patients now had a standardised care pathway that would improve the overall quality of the service.

The electronic dashboard has helped to keep all aspects of the care bundle easily accessible and bypasses the need for each individual in the team to remember every detail of the service that should be provided.

In conclusion, this project was felt to have helped to make a large improvement with the simple implementation of the electronic dashboard.

\section{References}

1. Schwab I, Nimmerjahn F. Intravenous immunoglobulin therapy: how does IgG modulate the immune system? ,Nature Reviews Immunology 13, 2013, Mar,176-189

2. Wimperis J, Lunn M, Jones A, Herriot R, Wood $P$, O'Shaughnessy $\mathrm{D}$, et al. Clinical guidelines for immunoglobulin use, 2nd Edition update, July 2011

3. Bayry J,Kazatchkine MD, Kaveri S. Nature Reviews Neurology 3, 2007 Mar, 120-121

\section{Declaration of interests}

Nothing to declare

\section{Acknowledgements}

Emma Donaldson 